\title{
Functional Outcome of Distal Radius Fractures Managed by Barzullah Working Classification
}

\author{
Younis Kamal ${ }^{1, *}$; Hayat Ahmad Khan ${ }^{1}$; Munir Farooq ${ }^{1}$; Naseemul Gani ${ }^{1}$; Ansar Ul Haq Lone \\ ; Adil Bashir Shah ${ }^{1}$; Irfan Ahmad Latto ${ }^{1}$; Mohammad Ashraf Khan ${ }^{1}$ \\ ${ }^{1}$ Post Graduate Department of Orthopedics, Bone and Joint Hospital, GMC Srinagar, Jammu and Kashmir, India \\ ${ }^{*}$ Corresponding author: Younis Kamal, Post Graduate Department of Orthopedics, Bone and Joint Hospital, GMC Srinagar, Jammu and Kashmir, India. Tel: +91-9906966960, E-mail: \\ kdryounis@gmail.com
}

Received: May 7, 2014; Revised: July 28, 2014; Accepted: December 28, 2014

\begin{abstract}
Background:Management of distal radius fractures (DRFs) is still controversial and maybe influenced by the initial fracture classification. Even though numerous classification systems have been proposed in this regard, the evaluation and management of this fracture has remained problematic.

Objectives: The purpose of this study was to evaluate the functional outcome of DRF managed on the basis of a new classification. This classification named as Barzullah Working Classification represents a modification of Melone classification, which is based on fracture stability.

Patients and Methods: A total of 310 DRFs of patients skeletally matured referred to a tertiary care hospital at a period of 18 months were classified as per the new classification system into four types; metaphyseal stable, metaphyseal unstable, radiocarpal stable, and radiocarpal unstable fractures. They were managed and followed over a mean period of $15.10 \pm 5.4$ months, and the results were recorded at the final follow-up.

Results: The mean age of the patients was $51.22 \pm 20.58$ years. Most of the patients were females $(n=189,64.19 \%)$. The minimal follow up was 6 months with a mean of $15.10 \pm 5.4$ months. Mean mayo wrist scores were $95 \pm 4,80 \pm 7.4,75 \pm 7.4$, and $70 \pm 6.9$, for stable metaphyseal fractures, unstable metaphyseal radial, stable radiocarpal fractures and unstable radiocarpal fractures, respectively. The overall mean mayo wrist functional score was $80.58 \pm 12.3$ (good results) at final follow up.

Conclusions: Various modalities of treatment used differentially in different types of DRFs based on the Barzullah Working Classification give good results in spite of conflicting literature.
\end{abstract}

Keywords: Osteoporotic Fractures; Classification; Fracture

\section{Background}

Distal radius fractures (DRFs) are the most common fractures encountered in orthopedic practice and account for $20 \%$ of all fractures seen in the emergency room (1). Following near anatomic close reduction and cast immobilization, various factors can cause secondary displacement of the fracture fragments, including shortening, angle of reduction (dorsal angulation), and articular congruence. These factors determine the treatment outcomes of DRFs. In young adults, the fractures are typically the result of high-energy injuries such as motor accidentsor fall from height. In contrast, most of the DRFs in the elderly occur from low-energy injuries such as fall from a standing height or on an outstretched hand $(2,3)$. Management of DRFs is still controversial and may be influenced by the initial fracture classification (4). Even though numerous classification systems have been proposed e.g., Frykman, Mayo, Melone, and AO, the evaluation and management of these fracture is yet con- troversial (5-8). Older classifications take into consideration several different parameters, such as the length of the radial styloid, dorsal angulation, and comminution of the dorsal cortex, but they overlook comminution of the volar cortex (9). We believe that the volar cortex and its comminution are of utmost importance for fracture stability and choice of treatment, as it behaves as the calcar of the distal radius $(10,11)$. The reliability and reproducibility rates of the AO, Frykman, Fernandez, and universal classification systems are insufficient and do not have advantages over each other (12). New classification systems are required to maintain a common language when defining DRFs (12).

\section{Objectives}

The purpose of this study was to evaluate the functional outcome of DRFs managed on the basis of a new,

Copyright (C) 2015, Kashan University of Medical Sciences. This is an open-access article distributed under the terms of the Creative Commons Attribution-NonCommercial 4.0 International License (http://creativecommons.org/licenses/by-nc/4.0/) which permits copy and redistribute the material just in noncommercial usages, provided the original work is properly cited. 
versatile, and comprehensive Barzullah Working Classification of DRFs found on fracture stability. The new Barzullah Working Classification is originally a modification of the Melone classification categorizing DRF into stable and unstable types depending upon the fracture pattern and loss of various stable corners of distal end radius. However, this classification fails to group pure metaphyseal fracture without intra-articular extension and is also difficult to follow and reproduce compared to our classification. Our classification is simple to remember and reproduce. It is also hierarchal, as the fracture complexity increases with advancing class of fracture. In addition, our new classification guides the management of a particular fracture type, and thus reduces the forthcoming loss of reduction and poor outcome of these fractures. It is a prognostic classification as well, as it can predict the likely outcome of a particular fracture type.

\section{Patients and Methods}

All patients with DRF were included in this study. They were referred to Emergency Department of Bone and Joint Surgery of Barzullah Hospital from May 2011 to November 2013. Patients were excluded from the study with the following criteria: less than 18 years of age, having compound fractures, DRF associated with a distal ulna fracture or associated with other injuries and patients refusing treatment as per protocol of the study. After taking routine posterioanterior and lateral radiographs of the wrist, the patients were divided as per the Barzullah Working Classification in the emergency department and were managed as per the set protocol of the study.

Distal radius fractures were divided by Barzullah Working Classification into metaphyseal fractures (I, Stable; II, Unstable)and radiocarpal fractures (I, Stable; II,Unstable). Criteria for unstable fracture are as follows:

1) Extensive dorsal comminution,

2) Fracture of volar buttress plate (volar calcar),

3) Fracture angulations $>20^{\circ}$,

4) Radial shortening $>5 \mathrm{~mm}$,

5) Articular step $>2 \mathrm{~mm}$,

6) DRUJ (Distal radial ulnar joint) incongruity,

7) Failure of conservative treatment,

All stable metaphyseal fractures were posted for closed reduction and short-arm cast under haematoma block or IV sedation. All unstable metaphyseal fractures were stabilized by transarticular external fixator under image intensifier or by open reduction/internal fixation (ORIF) in elective theatre. In all radiocarpal stable fractures, closed reduction and percutaneous pinning were done under image intensifier and short-arm cast was applied too. Radiocarpal unstable fractures, which include Barton's type fracture, high velocity, or fracture having intra-articular step more than $2 \mathrm{~mm}$ were preliminary close reduced and then sent for CT scan of the wrist.
Those fractures amenable to open reduction and internal fixation were posted for fixation in elective operative list. Highly comminuted fractures not amenable to ORIF were stabilized by transarticular external fixator. Patients with closed reduction, cast, and percutanous pinning with cast were discharged on the same day. Any fracture showing displacement at one or two weeks of follow-up were considered unstable and managed as unstable type of fracture. Sixteen patients lost to the follow-up and were excluded from the study. Patients were followed in the outpatient department in 1, 3 and 6 weeks. Around 6 weeks period, cast, K-wires, or external fixator were removed and the patient was sent for physiotherapy. In those patients with internal fixation, range-of-motion exercises were started on the second postoperative day. Radiological union was considered when a minimum of 3 cortices showed trabeculae crossing at the fracture site. Mayo wrist scoring was done at final follow-up.

\subsection{New Classification}

Distal radial fractures are classified according to the Barzullah Working Classification system into (Figure 1):

\subsubsection{Metaphyseal Fractures}

a) Stable fracture (extra-articular DRF with no cortical comminution).

b) Unstable fracture (extra-articular DRF with one or more unstable criteria).

\subsubsection{Radiocarpal Fractures}

a) Stable fracture (intra-articular DRF with no unstable fracture criteria).

b) Unstable fracture (intra-articular DRF like carpal fracture-dislocation, Barton fractures and Chauffeur fractures).

\section{Results}

A total of 310 patients (males $=111$, females $=199$ ) with mean age of $51.22 \pm 20$ year were included in this study. The minimal follow-up was 6 months with the mean of $15.10 \pm 5.4$ months. Sixteen patients lost to the follow-up and were excluded from the study. Patients' data regarding different classes with their mean age, male female ratio and their mean follow-up are presented in Table 1. Mean Mayo wrist score for the individual type of fracture was $95 \pm 4,80 \pm 7.4,75 \pm 7.4,70 \pm 6.9$ for stable metaphyseal fractures, unstable metaphyseal radial, stable radiocarpal fractures, and unstable radiocarpal fractures, respectively. The mean mayo wrist functional score was $80.58 \pm 12.3$ (good results) at final follow-up. Mean mayo wrist score at final follow-up is also given in Table 1. Table 2 shows various fracture types and different treatment modalities received. Mean time for radiological union was 10.25 weeks. 

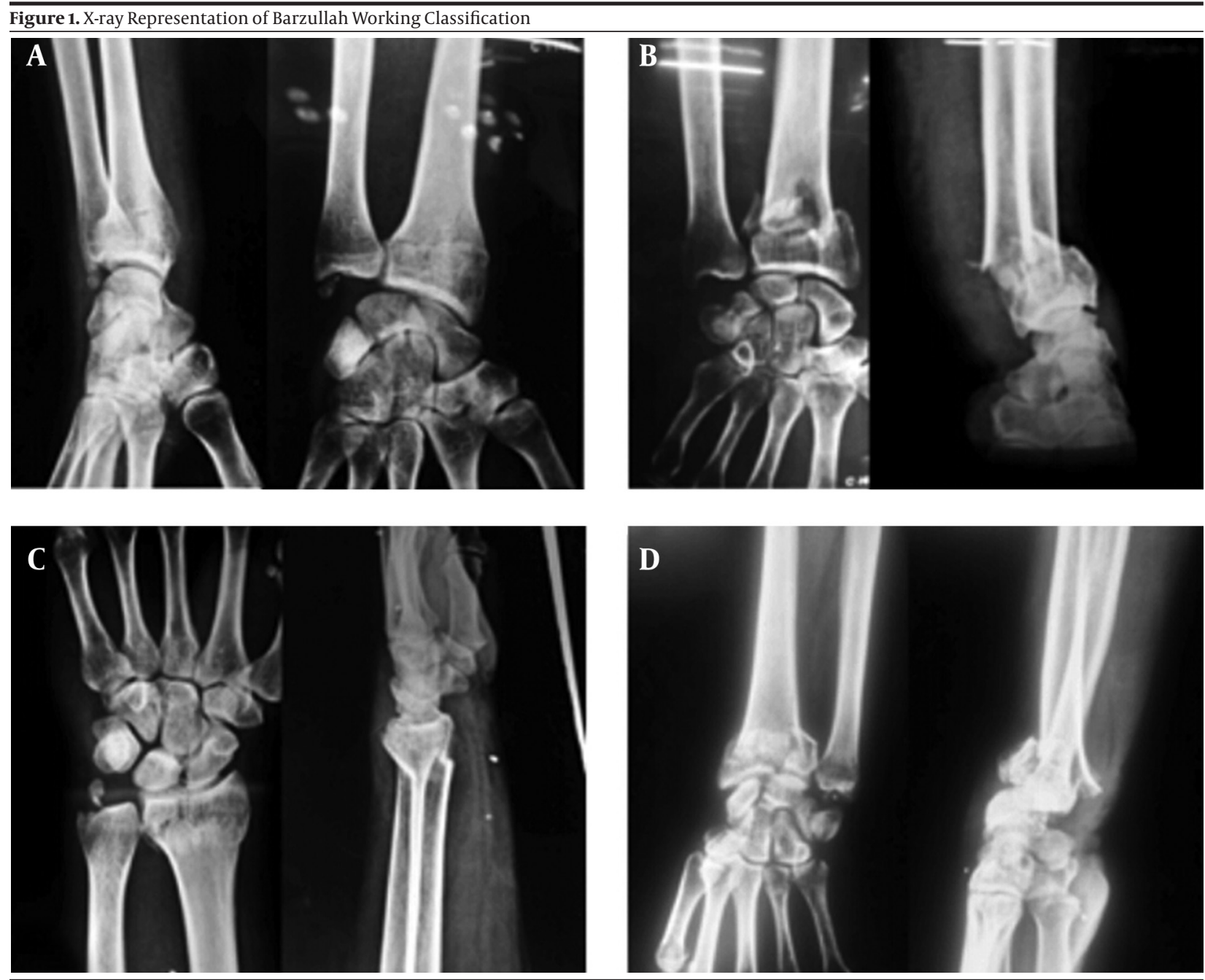

From Top left clockwise; Metaphyseal Stable, Metaphyseal Unstable, Radiocarpel Stable and Radiocarpel Unstable.

Table 1. Mean Mayo Wrist Score at Final Follow-Up ${ }^{\text {a }}$

\begin{tabular}{|c|c|c|c|c|c|c|c|}
\hline Fracture type & No. of patients & Average age, $y$ & Male & Female & $\begin{array}{c}\text { Treatment } \\
\text { received }\end{array}$ & $\begin{array}{c}\text { Mean follow } \\
\text { up, mo }\end{array}$ & $\begin{array}{c}\text { Mean mayo } \\
\text { wrist Score }\end{array}$ \\
\hline Metaphyseal stable & 130 & 59.3 & 41 & 89 & SAC & $14.83 \pm 5$ & $95 \pm 4$ \\
\hline Metaphyseal unstable & 73 & 48.7 & 27 & 46 & Ext. Fix. Or ORIF & $14.71 \pm 5$ & $80 \pm 7.4$ \\
\hline Radiocarpal stable & 82 & 43.5 & 24 & 58 & PCP, SAC & $15.52 \pm 5$ & $75 \pm 7.4$ \\
\hline Radiocarpal unstable & 25 & 37.9 & 19 & 6 & ORIF or Ext.Fix. & $13.96 \pm 6$ & $70 \pm 6.9$ \\
\hline Total & 310 & & 111 & 199 & & & \\
\hline
\end{tabular}

${ }^{\text {a }}$ Abbreviations: Ext. Fix, external fixator;ORIF, open reduction internal fixation; PCP, percutanous pinning; SAC, short arm cast.

There were 10 cases of complex regional pain syndrome I (CRPS I) which were managed by assurance and physiotherapy, out of them, 9 resolved completely, one patient was sent to psychiatric consultation. There were 22 cases with superficial infection and 7 cases with deep infection, all treated with antibiotics and daily dressing. Two external fixators were removed 2 weeks earlier and the patients were put on posterior splints for 2 weeks. Eleven patients complained of intermittent paresthesias over the volar aspect and managed by neurotropics. Complications detected in the external fixator were 2 metacarpal fractures managed conservatively by removing the pin and new pins were put in the third metacarpal. In 3 patients, injury to the cutaneous branch of the radial nerve occurred. One patient in the external fixator developed a compartmental syndrome (diagnosed by disproportionate pain, pain with finger extension) and fasciotomy of all of the compartments of the hand and forearm was performed to the patient. All of the complications had favorable resolution except the compartment syndrome that resulted in a claw hand with functional limitation (Figures 2 and 3). 
Kamal Yetal.

Table 2. Various Fracture Types and Different Treatment Modalities Received ${ }^{\mathrm{a}}$

\begin{tabular}{lcccccc}
\hline Fracture Type & No. of patients & SAC $(\mathbf{5} \pm \mathbf{2}$ wk) & PCP/SAC $(\mathbf{6} \pm \mathbf{1 w k})$ & Ext. Fix. $(\mathbf{6} \pm \mathbf{3}$ wk) & $\begin{array}{c}\text { ORIF } \\
\text { Radiological } \\
\text { union, wk }\end{array}$ \\
\hline Metaphysealstable & 130 & 130 & 0 & 0 & 0 & $8 \pm 3$ \\
Metaphyseal unstable & 73 & 0 & 39 & 25 & 9 & $11 \pm 4$ \\
Radiocarpal stable & 82 & 0 & 71 & 0 & 11 & $10 \pm 3$ \\
Radiocarpal unstable & 25 & 0 & 0 & 11 & 14 & $12 \pm 4$ \\
Total & 310 & 130 & 110 & 36 & 34 \\
\hline
\end{tabular}

a Abbreviations: Ext. Fix, external fixator; ORIF, open reduction internal fixation; PCP, percutanous pinning; SAC, short arm cast.
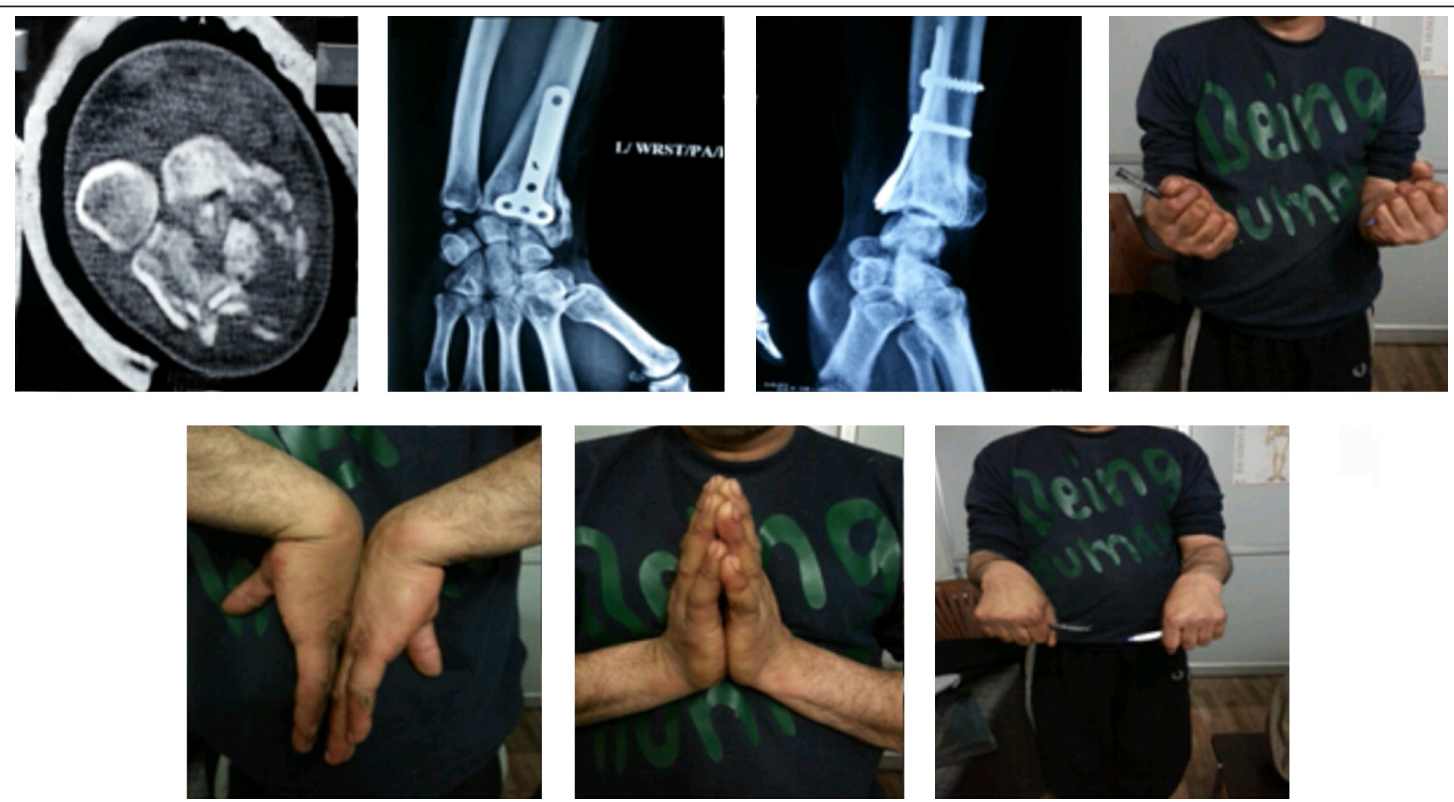

Figure 2. Case No. 1, CT Scan Union X-ray Range of Motion
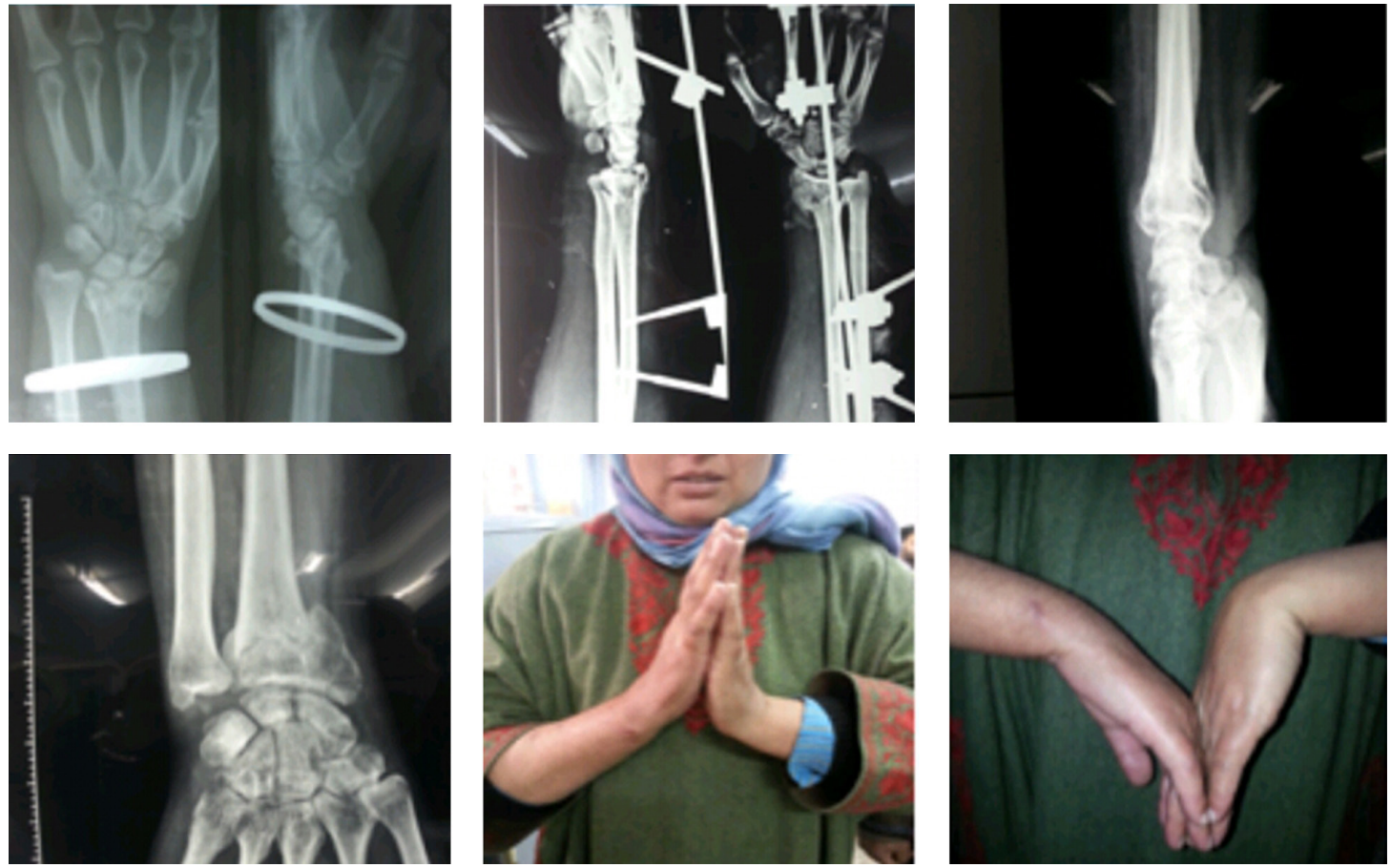

Figure 3. Case No. 2, Preoperative X-ray, Transarticular External Fixator X-ray Showing Progressive Union Rom at 3 Months 
Kamal Yet al.

\section{Discussion}

Management of DRF has been the most debatable topic among the orthopedic fraternity. Perhaps no other fracture has got so many eponyms over time. The resultant conflicting understanding of each eponym creates difficulty in assessing outcomes following treatment. To remedy this, several classification systems have been proposed. Some classifications seem to be more of an attempt to stress the significance of some features of the fracture rather than to provide a global approach. However some classifications have stood the test of time and are useful like Gartland and Werley (13), Frykman (5), Melone (6), Fernandez (7), and OTA/AO classifications (8). Illarramendi et al. have not recommended Frykman and AO classification for clinical use in view of their questionable reproducibility (14). Fernandez classification has the highest reliability rate and the universal classification has the lowest. None of the evaluated classifications could meet the expected reliability level. Frykman and Fernandez classifications were better in terms of reproducibility. However, none of them had good reproducibility rates (12).

At two international committee meetings during the fifth and sixth IFSSH Congresses, (May, 1992, Paris, France and July, 1995, Helsinki, Finland), Bone and Joint Committee reviewed each classification, but could not reach a consensus, much less unanimity, to support one of these classifications at the expense of the other (15). The characteristics agreed upon were as follows: 1) location, 2) configuration, 3) displacement, 4) ulnar styloid integrity, 5) distal radioulnar joint integrity, 6) stability, 7) associated injuries, and 8) bone mineralization.

From casting to arthroscopic assisted reduction, all treatment modalities have been tried. Sir Abraham Colles first described the deformities of distal end radius fractures many years before the advent of X-rays. Since then, a lot of modifications in the management of DRF have taken places, which include closed reduction with casting, percutaneous pinning, intrafocal pinning, external fixation with ligamentotaxis, minimal open to open reduction and internal fixation with various modern gadgets. Even the fragment specific fracture fixation has been tried with good results. Karimi et al. in his study has shown good results in torus fractures in patients less than 17 years of age using removable wrist splint. He, however, studied only the patients of less than 17 (16).Bahari-Kashani et al. (2003) studied the intra-articular distal end radius fractures managed by pin and plaster or plating. They concluded that functional scoring, radiological indices, grip strength, and supination pronation were better in the plating group (17). Mardani et al. compared the closed reduction with long-arm cast to closed reduction with PCP and found the latter as better option in terms of finger stiffness, but found some pin tract infections, which resolved uneventfully (18).
Melone classification grouped DRF into stable and unstable fractures. But this classification fails to categorize pure metaphyseal fracture without intra-articular extension, whereas our new classification includes all DRFs, extra-articular as well as intra-articular in a hierarchal way with increase in fracture complexity with each advancing type. Our classification is simple to remember and reproduce. In addition, our new classification guides regarding the management of a particular fracture type, thus reducing the chance of loss of reduction and poor outcome of these fractures. It is a prognostic classification too as we can predict the likely outcome of a particular fracture pattern.

The difference between a stable and unstable metaphysealorradiocarpal fracture, depends upon various radiological dimensions, which has been proven to affect the outcome of the DRF. Cooney et al. suggested that fractures with severe dorsal comminution and dorsal angulation greater than $20^{\circ}$ have a significant chance of re-displacement after reduction (19). Vaughan et al. emphasized on the importance of dorsal angulation and radial shortening (20). Instability is defined by re-displacement of the fracture site after manipulating into anatomic position. We believe that the volar cortex and its comminution is of uttermost importance for fracture stability and choice of treatment, as it behaves as the calcar of the distal radius $(9,10)$. Lafontaine et al. extended these concepts and suggested that dorsal angulation more than 20 degrees, presence of ulnar fracture, patients age more than 60 years, dorsal comminution, and intra-articular fracture lineare risk factors for fracture instability (21).

Various classifications of DRFs have been proposed in the past two centuries. Fracture eponyms pay tribute to those who initiated the process, including Pouteau, Colles, Barton, Goyrand, and Smith. With this foundation, many investigators progressively contributed to the breadth and depth of understanding of DRFs based on fracture attributes and severity. Each method of classification had its champions, who touted its strengths, but there were always critics who identified weaknesses as well. The present study is in continuity to evaluate and understand further the comprehensive management of distal fractures in more global and simple way. This study investigated the functional outcome of 310 DRFs managed in line with standard treatment but in a new and simple way. Small sample size and short follow-up are the limitations of this study to draw any definite conclusion. Further studies are needed to check credibility of the new classification in orthopedic practices. Various modalities of treatment used differentially in different types of DRFs based on the Barzullah Working Classification give good results in spite of conflicting literature.

\section{Acknowledgements}

We would like to thank Dr Snobar Gul, Dr Nazia Hassan, 
and Dr Hina Jeelani for their support during the initialdrafting and subsequent revisionof this paper.

\section{Authors' Contributions}

Study concept and design: Dr Younis Kamal, Dr Hayat Ahmad Khan and Munir Farooq; Acquisition of data: Dr Hayat Ahmad Khan,Younis Kamal, Naseemul Gani, and Ansarul haq Lone; Analysis and interpretation of data: Younis kamal, Hayat Ahmad khan, and Nassemul Gani; Drafting of the manuscript: Adil Bashir shah, Irfan Ahmad Latto, Mohammad Ashraf Khan , Hayat ahmad Khan, and Younis Kamal ; Critical revision of the manuscript for important intellectual content: Dr Naseemul Gani, Hayat Ahmad Khan, Younis Kamal, Munir farooq;and Study supervision: Munir Farooq, Younis Kamal, Hayat Ahmad Khan.

\section{References}

1. van Staa TP, Dennison EM, Leufkens HG, Cooper C. Epidemiology of fractures in England and Wales. Bone. 2001;29(6):517-22.

2. Nesbitt KS, Failla JM, Les C. Assessment of instability factors in adult distal radius fractures. J Hand Surg Am. 2004;29(6):1128-38.

3. Diaz-Garcia RJ, Oda T, Shauver MJ, Chung KC. A systematic review of outcomes and complications of treating unstable distal radius fractures in the elderly. JHand Surg Am. 2011;36(5):824-35 e2.

4. Handoll HH, Madhok R. Surgical interventions for treating distal radial fractures in adults. Cochrane Database Syst Rev 2003(3):CD003209.

5. Frykman G. Fracture of the distal radius including sequelae-shoulder-hand-finger syndrome, disturbance in the distal radio-ulnar joint and impairment of nerve function. A clinical and experimental study. Acta Orthop Scand. 1967:Suppl 108:3+.

6. Melone CJ. Open treatment for displaced articular fractures of the distal radius. Clin Orthop Relat Res. 1986(202):103-11.

7. Fernandez DL, Jupiter JB. Fractures of the Distal Radius.New York: Springer Verlag; 1995.
8. Müller ME, Koch P, Nazarian S, Schatzker J. The Comprehensive Classification of Fracture of Long Bones.Berlin: Springer Verlag; 1990.

9. Older TM, Stabler EV, Cassebaum WH. Colles Fracture: Evaluation and Selection of Therapy.J Trauma. 1965;5:469-76.

10. Dhillon SS, Kumar AJ, Sadaiyyappan V, Bassi RS, Shanahan D, Deshmukh SC. Anatomical study comparing the thickness of the volar and dorsal cortex of cadaveric adult distal radii using digital photography. Arch Orthop Trauma Surg. 2007; 127(10):975-7.

11. Mackenney PJ, McQueen MM, Elton R. Prediction of instability in distal radial fractures.J Bone Joint Surg Am. 2006;88(9):1944-51.

12. Kucuk L, Kumbaraci M, Gunay H, Karapinar L, Ozdemir O. Reliability and reproducibility of classifications for distal radius fractures. Acta Orthop Traumatol Turc. 2013;47(3):153-7.

13. Gartland JJ, Werley CW. Evaluation of healed Colles' fractures. $J$ Bone Joint Surg Am. 1951;33-A(4):895-907.

14. Illarramendi A, Gonzalez Della Valle A, Segal E, De Carli P, Maignon G, Gallucci G. Evaluation of simplified Frykman and AO classifications of fractures of the distal radius. Assessment of interobserver and intraobserver agreement. Int Orthop. 1998;22(2):111-5.

15. IFSSH BONE AND JOINT COMMITTEE.. How To Classify Distal Radial Fractures. 1998. Available from: http://www.eradius.com/IFSSH_ Classification_D1.htm.

16. Karimi Mobarakeh M, Nemati A, Noktesanj R, Fallahi A, Safari S Application of removable wrist splint in the management of distal forearm torus fractures. Trauma Mon. 2013;17(4):370-2.

17. Bahari-Kashani M, Taraz-Jamshidy MH, Rahimi H, Ashraf $\mathrm{H}$ Mirkazemy M, Fatehi A, et al. Outcomes of pin and plaster versus locking plate in distal radius intraarticular fractures. Trauma Mon. 2013;17(4):380-5.

18. Mardani KM, Asadi K, Hashemi Motlagh K, Shakiba M. Distal Radius Fracture, a Comparison between Closed Reduction and Long Arm Cast Vs. Closed Reduction and Percutaneous Pinning and Short Arm Cast. Shiraz E Med J. 2011;12(3).

19. Cooney W3, Linscheid RL, Dobyns JH. External pin fixation for unstable Colles' fractures.J Bone Joint Surg Am. 1979;61(6A):840-5.

20. Vaughan PA, Lui SM, Harrington IJ, Maistrelli GL. Treatment of unstable fractures of the distal radius by external fixation.J Bone Joint Surg Br. 1985;67(3):385-9.

21. Lafontaine M, Hardy D, Delince P. Stability assessment of distal radius fractures. Injury. 1989;20(4):208-10. 